\title{
Risk aggregation in Solvency II through recursive log-normals
}

\author{
Erik Bølviken and Montserrat Guillen* \\ University of Oslo University of Barcelona
}

December 21, 2016

\begin{abstract}
It is argued that the accuracy of risk aggregation in Solvency II can be improved by updating skewness recursively. A simple scheme based on the log-normal distribution is developed and shown to be superior to the standard formula and to adjustments of the Cornish-Fisher type. The method handles tail-dependence if a simple Monte Carlo step is included. A hierarchical Clayton copula is constructed and used to confirm the accuracy of the log-normal approximation and to demonstrate the importance of including tail-dependence. Arguably a log-normal scheme makes the logic in Solvency II consistent, but many other distributions might be used as vehicle, a topic that may deserve further study.
\end{abstract}

JEL codes: G22, G28.

Key words and phrases

Clayton copula, Cornish-Fisher, moment matching, recursive skewness, standard formula, sum of lognormals.

\footnotetext{
*Corresponding author: mguillen@ub.edu Dept.Econometrics, Riskcenter-IREA, University of Barcelona, Diagonal, 690, E-08034 Barcelona, Spain. Phone: +34934037039.
} 


\section{Introduction}

Solvency II insurance regulation addresses uncertainty by first subtracting a so-called 'best estimate' so that company risk becomes a sum of zero-mean random variables organized as a hierarchy. At the top level there are insurance risk, market risk, credit risk and operational risk as in Figure 1 with components and sub-components underneath in structures that might in practice be much more complex than shown. The risk is controlled and supervised through the so-called solvency capital requirement (SCR) which is the $99.5 \%$ percentile of all net obligations for the coming year. Determining it is no easy task, certainly not computationally, but nor in terms of modelling with so many parameters with limited data to hang estimates on. A regulatory body must also strike a balance between accuracy and simplicity. Monte Carlo might appear an obvious computational tool, but considerable modelling effort and computer programming would be needed for a full bottom-up model which may be prohibitively costly for not-so-large companies. Perhaps it would also be inconvenient for regulators to supervise, and at any rate the solution adopted in Solvency II is different and much simpler.

The idea is to allocate SCR's (or $99.5 \%$ percentiles) to all risk variables individually and accumulate them to a value for the entire company. To clarify the issue consider the simplified skeleton of a non-life insurance company in Figure 1. It is a simplified extract of the Solvency II flow-chart in EIOPA(2014) and will be used for illustrations later. The top SCR encompassing all company operations branches out into components and sub-components. Those with no offshoots under them, for example operational, lapse, credit risk and others are input to the scheme and have their SCR's specified as laid down by EU legislation in the Commission Delegated Regulation (2015) or by internal quantification. These assessments are then merged to a new value one level above, and in this manner the tree is climbed until the top for the entire company has been reached. The question is how the 
rule of aggregation should be constructed. Solvency II has opted for the so-called standard formula which is exact for Gaussian systems, but in insurance and finance this condition is not too plausible due to the existence of extremes and asymmetry; see Pfeifer and Strassburger (2009). The purpose of this article is to promote schemes with higher accuracy while keeping things simple.

The most obvious objection to the Gaussian model is the symmetry forced upon all marginal distributions. This may not be very inaccurate for market risk extending over one year, see Ch 15 in Sandström (2011), but it is wide off the mark for all those variables in insurance with heavy right tails. Can skewness corrections be introduced in some way? The issue was taken up by Sandström (2007) who used the Cornish-Fisher improvement of Gaussian approximations, and he was able to demonstrate that skewness can be updated recursively and jointly with the SCR updates themselves. However, there are other ways this can be done, and the problem deserves more research. One possibility which is developed and examined below, is the use of log-normal distributions with a parameter capturing skewness. This contributes an enhanced degree of consistency since the log-normal model as motivating factor is present everywhere in Solvency II, and the added complexity compared to the standard formula is one update more. The skewness recursion differs from that in Sandström (2007) and is, as we shall argue, a more effective one.

But why not other families of heavy-tailed distributions? Closed expressions for the update formulae as in Section 2 are no longer possible, but it can be done through numerics; see Section 3 . We might go even further. One of the referees has pointed out that the most accurate of all would be to assign different types of distributions to different variables, and for example to consider the log skew normal (Eling, 2012). The approach developed below handles even this, yet it is open to question how far down this road it is practical to go. The parameters in heavy-tailed variables are often highly 
uncertain due to lack of data whatever the family of distributions selected, and a regulator must also guard against making schemes too complex.

Then there is a second question of how covariation should be modelled. The standard Gaussian model makes use of ordinary correlations which is a serious limitation unable to capture tail dependence. Papers addressing the issue in Solvency II are Pfeifer and Strassburger (2008), Savelli and Clemente (2011), Bermudez, Ferri and Guillen (2013) and Alm (2015) who investigated the consequences for the accuracy of the standard formula and proposed other ways to proceed. The log-normal approach can be fortified in this direction through copula constructions at the expense of using a small dose of Monte Carlo in the skewness update. As pointed out in Savelli and Clemente (2011) we are now entering the realm of hierarchical copulas with highly unequal degree of dependence for different groups of variables. To evaluate the accuracy of the log-normal aggregation method in the example in Figure 1 a bottom-up model is needed. The approach through nested Archimedeans in McNeil (2008) proved convenient and shall enable us to indicate the significance of using copulas rather than correlations to describe dependencies between variables.

\section{Solvency capital recursions}

\subsection{The standard formula}

The recursive step in the Solvency II aggregation scheme can be expressed as follows. Let $X_{1}, \ldots, X_{n}$ be dependent random variables with mean 0 , standard deviations $\sigma_{1}, \ldots, \sigma_{n}$ and $1-\epsilon$ percentiles $C_{1}, \ldots, C_{n}$. The reason for zero mean variables is that the expected in Solvency II has already has been taken through the so-called 'best estimate', as indicated earlier. Introduce

$$
S=X_{1}+\ldots+X_{n}
$$




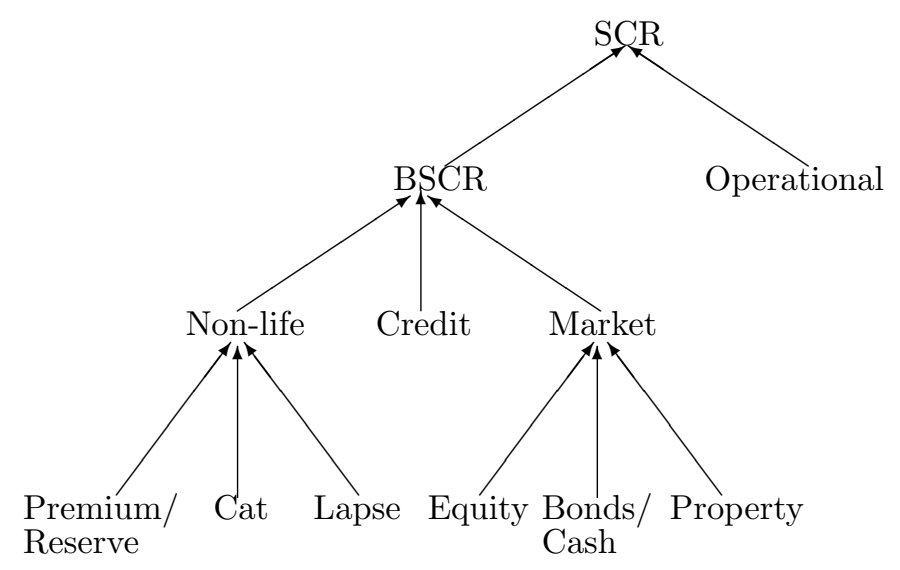

Figure 1 Solvency II representation of the risk components of a simplified insurance company.

and write $\sigma_{s}$ and $C_{s}$ for the standard deviation and $1-\epsilon$ percentile of this sum. The issue is then to compute $C_{s}$ from $C_{1}, \ldots, C_{n}$ which is achieved through the standard formula

$$
C_{s}=\left(\sum_{i=1}^{n} \sum_{j=1}^{n} \rho_{i j} C_{i} C_{j}\right)^{1 / 2}
$$

with $\rho_{i j}=\operatorname{cor}\left(X_{i}, X_{j}\right)$, see the Commission Delegated Regulation (2015). In the Gaussian case $C_{i}=\sigma_{i} \phi_{\epsilon}$ with $\phi_{\epsilon}$ the $1-\epsilon$ percentile of the standard normal, and the ordinary variance/covariance formula in (4) left shows that the method is exact for Gaussian systems.

It can be used recursively. Let $S_{1}, \ldots, S_{m}$ be sums similar to that in (1), and suppose their $1-\epsilon$ percentiles $C_{s 1}, \ldots, C_{s m}$ have been obtained by $m$ different applications of the rule in (2). The $1-\epsilon$ percentile of $S_{1}+\ldots+S_{m}$ may then be calculated by the same method provided the correlations of $S_{1}, \ldots, S_{m}$ have been specified which is precisely what is offered in the Commission Delegated Regulation (2015). Repeated applications of this technique while going upwards through the branches of Figure 1 may proceed until the top that applies to the entire company and all its operations has been reached. Initialization of the scheme when the input SCR's are specified, is typically carried out independently. Indeed on this point the Commission Delegated Regulation (2015) often refers to 
log-normal rather than Gaussian distributions.

\subsection{A Cornish-Fisher method}

The aggregation rule in Section 2.1 is unlikely to be very accurate since skewness, often important in insurance, is neglected. A possible remedy is to draw on the Cornish-Fisher approximation in statistics. The idea which is due to Sandström (2007), makes use of the skewness coeffcients $\gamma_{1}, \ldots, \gamma_{n}$ of the variables $X_{1}, \ldots, X_{n}$ and starts by adding the Cornish-Fisher term so that now

$$
C_{i}=\sigma_{i}\left\{\phi_{\epsilon}+\gamma_{i}\left(\phi_{\epsilon}^{2}-1\right) / 6\right\}, \quad i=1, \ldots, n
$$

which are to be converted into an assessment $C_{s}$ of the solvency capital of $S$ one level higher up. This is achieved by evaluating the standard deviations $\sigma_{s}$ and skewness coefficient $\gamma_{s}$ of $S$ through

$$
\sigma_{s}=\left(\sum_{i=1}^{n} \sum_{j=1}^{n} \rho_{i j} \sigma_{i} \sigma_{j}\right)^{1 / 2} \quad \text { and } \quad \gamma_{s}=\frac{\gamma_{1} C_{1}+\ldots+\gamma_{n} C_{n}}{C_{1}+\ldots C_{n}}
$$

and when $\sigma_{s}$ and $\gamma_{s}$ replace $\sigma_{i}$ and $\gamma_{i}$ in (3), we arrive at $C_{s}$. This again sets up a recursion. If $\sigma_{s 1}, \ldots, \sigma_{s m}, \gamma_{s 1}, \ldots, \gamma_{s m}$ and $C_{s 1}, \ldots, C_{s m}$ have been obtained as standard deviation, skewness and solvency capital for $S_{1}, \ldots, S_{m}$, then the same quantities can be found for $S_{1}+\ldots+S_{m}$.

The method is presented and motivated differently in Sandström (2007), but it is apart from this exactly the same, see also Savelli and Clemente (2011) for a version that is a variation of Sandström's. A point for discussion is the skewness aggregation. Does it really make sense to evaluate $\gamma_{s}$ as a weighted average of $\gamma_{1}, \ldots, \gamma_{n}$ as in (4) right when we know by the central limit theorem that skewness has a tendency to go down when random variables are added? Or suppose $\gamma_{1}=\ldots=\gamma_{n}=\gamma$ with $X_{1}, \ldots, X_{n}$ independent. Then $\gamma_{s}=\gamma / \sqrt{n}$, very far below $\gamma_{s}=\gamma$ returned by (4) right. This estimate has in our opinion a strong tendency to over-estimate skewness. The consequences will be evaluated in Section 4. 


\subsection{A log-normal solution}

In reality a non-parametric approach to skewness correction, as in the preceding section, is doomed to failure when $X_{1}, \ldots, X_{n}$ are dependent. The expression (8) below shows that the skewness $\gamma_{s}$ depends on cross-moments such as $E\left(X_{i}^{2} X_{j}\right)$ and $E\left(X_{i} X_{j} X_{k}\right)$ which are out of reach in any simple manner without imposing distributions on $X_{1}, \ldots, X_{n}$. If that is done, $\sigma_{s}$ and $\gamma_{s}$ can be selected so that they match those that can be calculated from $X_{1}, \ldots, X_{n}$, and this offers hope of improving the accuracy since the shape of distributions now is much better preserved when moving from one level to another.

There are many ways to proceed from here (see Section 3), but an obvious first choice is log-normals which are envisaged everywhere in the Solvency II regime. A useful form of the model is

$$
X_{i}=\sigma_{i} \frac{e^{-\tau_{i}^{2} / 2+\tau_{i} \varepsilon_{i}}-1}{\left(e^{\tau_{i}^{2}}-1\right)^{1 / 2}} \quad \text { with } \quad \varepsilon_{i} \sim N(0,1) .
$$

Here $\tau_{i}$ a parameter capturing skewness. Note that $X_{i} \rightarrow \sigma_{i} \varepsilon_{i}$ as $\tau_{i} \rightarrow 0$ by an elementary application of l'Hopital's rule, and the normal distribution is the special case $\tau_{i}=0$. The model is further motivated by the properties summarized in the following proposition:

Proposition 1 For the model in (5)

$$
\begin{aligned}
& E\left(X_{i}\right)=0, \quad \operatorname{sd}\left(X_{i}\right)=\sigma_{i}, \quad \gamma_{i}=\left(e^{\tau_{i}^{2}}+2\right) \sqrt{e^{\tau_{i}^{2}}-1} \\
& \tau_{i}=\sqrt{\log \left(A_{i}+A_{i}^{-1}-1\right)} \quad \text { where } \quad A_{i}=\left(1+\gamma_{i}^{2} / 2-\sqrt{\gamma_{i}^{4} / 4+\gamma_{i}^{2}}\right)^{1 / 3} .
\end{aligned}
$$

Proof Recall that $e^{-\tau_{i}^{2} / 2+\tau_{i} \varepsilon_{i}}$ has mean 1 and variance $e^{\tau_{i}^{2}}-1$, and the expressions for $E\left(X_{i}\right)$ and $\operatorname{sd}\left(X_{i}\right)$ follow. The skewness $\gamma_{i}$ on the right in (6) is the formula for the skewness of log-normal variables, see Bølviken (2014), p 319, and it determines $\tau_{i}$ from $\gamma_{i}$ though a cubic equation for $e^{\tau_{i}^{2}}$. There 
is a unique real solution which is (7).

A main point in Proposition 1 is that it is simple to pass from $\gamma_{i}$ to $\tau_{i}$ and back, but the exact distribution of the sum $S$ is complicated, and must be approximated. There is a considerable literature on this extending over several branches of applied mathematics. The most common approach, followed below, is to use another log-normal with correct standard deviation and skewness. Such moment matching goes back to Wilkinson (1934), and is in communication theory known as the Fenton-Wilkinson approach after Fenton (1960). There is an error bound in Berggren (2005). The early contributions dealt with independent variables only, but extensions to correlated ones can be found in Dufresne (2004) and Henriksen (2008) with efficient Monte Carlo for the extreme tails in Asmussen, Blanchet, Juneja and Rojas-Nandyapa (2011) and a limit theorem in Beaulieu (2012). A completely different approximation based on moment generating functions was developed in Mehta, Wu, Molisch and Zhang (2007), and this is an alternative to the line pursued here. Some authors, notably Beaulieu and Xie (2004), Zhang and Song (2008) and Senaratne and Tellambura (2009) argue that other distributions than the log-normal provide better fit, but that is not attractive in our situation of multiple, recursive applications of the same method.

Moment matching means that we have to determine the third order moment $E\left(S^{3}\right)$ and convert it to skewness through $\gamma_{s}=E\left(S^{3}\right) / \sigma_{s}^{3}$. Elementary calculations show that

$$
E\left(S^{3}\right)=\sum_{i} E\left(X_{i}^{3}\right)+3 \sum_{i \neq j} E\left(X_{i}^{2} X_{j}\right)+6 \sum_{i<j<k} E\left(X_{i} X_{j} X_{k}\right)
$$

where closed expressions for the cross-moments are available when we have imposed correlations $\rho_{i j}=\operatorname{cor}\left(X_{i}, X_{j}\right)$ as in Solvency II which yields: 
Proposition 2. The skewness $\gamma_{s}$ of $S$ under the log-normal distribution is

$$
\begin{aligned}
\gamma_{s}=\sum_{i} \alpha_{i}^{3}\left(e^{\tau_{i}^{2}}+2\right)\left(e^{\tau_{i}^{2}}-1\right)^{2} & +3 \sum_{i \neq j} \alpha_{i}^{2} \alpha_{j}\left\{e^{\tau_{i}^{2}}\left(\beta_{i j}^{2}-1\right)-2\left(\beta_{i j}-1\right)\right\} \\
& +6 \sum_{i<j<k} \alpha_{i} \alpha_{j} \alpha_{k}\left\{\beta_{i j} \beta_{i k} \beta_{j k}-\beta_{i}-\beta_{j}-\beta_{k}+2\right\} .
\end{aligned}
$$

where

$$
\alpha_{i}=\frac{\sigma_{i}}{\sigma_{s} \sqrt{e^{\tau_{i}^{2}}-1}}, \quad \text { and } \quad \beta_{i j}=1+\rho_{i j} \sqrt{\left(e^{\tau_{i}^{2}}-1\right)\left(e^{\tau_{j}^{2}}-1\right)}
$$

Proof The detailed calculations can be found in Appendix A.

Proposition 2 produces the following method. Start at $\sigma_{1}, \ldots, \sigma_{n}$ and $\gamma_{1}, \ldots, \gamma_{n}$ and convert the second sequence to $\tau_{1}, \ldots, \tau_{n}$ by $(7)$. Now $(4)$ left and $(9,10)$ yield $\sigma_{s}$ and $\gamma_{s}$ with the corresponding $\tau_{s}$ obtained by another application of $(7)$ so that by (5)

$$
C_{s}=\sigma_{s} \frac{e^{-\tau_{s}^{2} / 2+\tau_{s} \phi_{\epsilon}}-1}{\left(e^{\tau_{s}^{2}}-1\right)^{1 / 2}}
$$

The scheme has a more complicated skewness update than Sandtröm's method, but it is easily programmed and recursive in the same way as the others.

\section{General constructions}

Other models than the log-normal can be used as vehicle for aggregation rules of this type. Let $F\left(x ; \tau_{i}\right)$ be a distribution function with mean 0 , standard deviation 1 , depending on some shape parameter $\tau_{i}$. With $F^{-1}\left(u ; \tau_{i}\right)$ its percentile function consider

$$
X_{i}=\sigma_{i} F^{-1}\left(U_{i} ; \tau_{i}\right), \quad i=1, \ldots, n
$$

where $U_{1}, \ldots, U_{n}$ are uniforms. This makes $F\left(x ; \tau_{i}\right)$ the distribution function of $X_{i} / \sigma_{i}$, and the direct extension of (5) is to let $U_{i}=\Phi\left(\epsilon_{i}\right)$ with $\Phi$ the standard normal integral. Correlations in $\varepsilon_{1}, \ldots, \varepsilon_{n}$ take 
care of dependency as before. The scheme can be developed for the Gamma, inverse Gaussian and Burr families, all with skewness varying over the entire positive, real line and with a links between skewness $\gamma_{i}$ and shape $\tau_{i}$. Closed expressions for $\gamma_{s}$ as in Section 2.3 are not possible, but that can be handled by Monte Carlo as in (14) below, and moment matching determines $\tau_{s}$ for the next round of the recursion.

One potential weakness in transforming Gaussians to variables with fat upper tails in this way is that it forces a specific tail dependence on the model with correlations becoming smaller at the right tail than on average. If this is considered undesirable, it can be corrected for by imposing a copula on $U_{1}, \ldots, U_{n}$ in (12). In the log-normal case the model (5) now becomes

$$
X_{i}=\sigma_{i} \frac{e^{-\tau_{i}^{2} / 2+\tau_{i} \Phi^{-1}\left(U_{i}\right)}-1}{\left(e^{\tau_{i}^{2}}-1\right)^{1 / 2}}, \quad i=1, \ldots, n
$$

where $\Phi^{-1}$ is the percentile function of the standard normal. The method is as in Section 2.3 except for the skewness update. Closed expressions for the cross-moments in (8) are no longer possible. Numerical integration is a possibility, but simulation is simpler in our view. If $\left(U_{1 l}^{*}, \ldots, U_{n l}^{*}\right)$ for $l=1, \ldots, N$ are Monte Carlo realizations of $\left(U_{1}, \ldots, U_{n}\right)$, then:

Proposition 3. Under the log-normal copula model (13) or the more general model (12) an approximation of the skewness of $S$ is

$$
\gamma_{s}=\frac{1}{\sigma_{s}^{3} N} \sum_{l=1}^{N}\left(\sum_{i}\left(X_{i l}^{*}\right)^{3}+3 \sum_{i \neq j}\left(X_{i l}^{*}\right)^{2} X_{j l}^{*}+6 \sum_{i<j<k} X_{i l}^{*} X_{j l}^{*} X_{k l}^{*}\right)
$$

where for the log-normal

$$
X_{i l}^{*}=\sigma_{i} \frac{e^{-\tau_{i}^{2} / 2+\tau_{i} \Phi^{-1}\left(U_{i l}^{*}\right)}-1}{\left(e^{\tau_{i}^{2}}-1\right)^{1 / 2}} \quad \text { or in case of }(12) \quad X_{i l}^{*}=\sigma_{i} F^{-1}\left(U_{i l}^{*} ; \tau_{i}\right) .
$$

Proof. Follows immediately from (8) like in Proposition 2. 
The approximation in Proposition 3 becomes exact as $N \rightarrow \infty$, but high Monte Carlo accuracy isn't necessary, and $N=1000$ or even $N=100$ simulations might be enough.

\section{Example: The non-life module in Solvency II}

\subsection{Dependence through correlations}

The first example is the non-life module in Figure 1 with 'Premium/Reserve' as variable $X_{1}$, large claims contracts ('Cat' which is $X_{2}$ ) and lapse risk ('Lapse' or $X_{3}$ ). The SCR's of Premium/Reserve and Cat are in practice computed from numerous sub-risks, but they are here log-normal percentiles. Skewness coefficients for Premium/Reserve and Lapse were selected as $\gamma_{1}=\gamma_{3}=0.15$ which means that the $99.5 \%$ percentiles are around 3 times the standard deviation, a frequent assumption in EIOPA (2014). For catastrophe risk skewness $\gamma_{2}$ is in practice much larger and is varied below. Standard

deviations of the three variables are those in Scenario A in Table 1, and correlations are as in EIOPA (2014); i.e. Premium/Reserve and Cat have correlation 0.25 with Lapse independent of the two others.

Solvency capital requirements have been plotted in Figure 2 left against the Cat skewness coefficient which was varied between $\gamma_{2}=0$ and $\gamma_{2}=6$. Note that $\gamma_{2}=6$ corresponds to the log-normal parameter $\tau_{2} \approx 1$ which yields a distribution with quite heavy tails. Methods examined are the log-normal and Cornish-Fisher approximations ('Logn') and ('CoFi') and two versions of the standard formula denoted 'Solvency II' and 'Gaussian' which differ in their input SCR's. 'Solvency II' took log-normal percentiles as $C_{i}$ (which imitates how Solvency II is used in practice), and 'Gaussian' normal ones. Approximately exact values based on one million Monte Carlo simulations have been appended as 'Exact'.

All methods are fairy accurate when skewness is small, but 'Gaussian' (for which skewness is not 
Figure 2 The solvency capital requirement against catastrophe skewness (left) and against a common skewness (right) when dependency is described by correlations. Note: Vertical axis scales differ.

taken into account at all) quickly under-estimates as $\gamma_{2}$ increases. The log-normal approximation follows the exact one with a maximum, conservative error about $8 \%$ when $\gamma_{2}=6$. The standard formula, also conservative is less accurate while the Cornish-Fisher method is far too high (which is caused by the skewness estimate being twice as large as it should). The experiment has been redone on the right in Figure 2 with common skewness $\gamma_{1}=\gamma_{2}=\gamma_{3}=\gamma$ for all three variables and with $\gamma$ varying between 0 and 6 . The log-normal method now follows the exact one closely, 'Solvency II' is also accurate whereas errors in 'Gaussian' and Cornish-Fisher are large.

\subsection{Dependence through the Clayton copula}

To what extent does the accuracy of log-normal solvency capital aggregation stand up when linear correlations are replaced by other mathematical descriptions of dependence? The illustration in Figure 3 is based on the same assumptions as in Section 4.1 except for Premium/Reserve and Cat now being related through a Clayton copula. A useful form of it is Marshall-Olkin mixing with $U_{1}$ and $U_{2}$ in (15) 
Figure 3 The solvency capital requirement against catastrophe skewness (left) and against a common skewness (right) under Clayton dependency. Note: Vertical axis scales differ.

specified as

$$
U_{i}=\left(1-\theta \frac{\log \left(V_{i}\right)}{Z}\right)^{-1 / \theta}, \quad i=1,2
$$

where $V_{1}$ and $V_{2}$ are independent and uniform and $Z$ Gamma-distributed with mean 1 and shape $1 / \theta$; consult Chapter 6 in Bølviken (2014) for the construction. Correlation between Premium/Reserve and Cat remains at 0.25 approximately if $\theta=0.326$ which was used for the experiments.

Methods examined in Figure 3 are the log-normal approximation in Section 2.3 and the alternative one in Section 3 based on the true copula with skewness at the next level determined by one million simulations (much more than needed). Nearly exact evaluations of solvency capital were produced by Monte Carlo (one million simulations). These assessments are plotted against the Cat skewness on the left and a common skewness value for all three variables on the right. The log-normal approximation based on the correct model is again accurate. Clayton dependence has real impact on the results even in a situation with such moderate dependency between variables. The method in Section 2.3 with the 
correct correlation, but without tail dependency taken into account, under-estimates $10-12 \%$ on the left and $15-18 \%$ on the right.

\section{A simplified Solvency II insurance company}

The second example is the Solvency II company in Figure 1 with credit and market risk added the non-life module in Section 3. The sum of these three components is the basic solvency capital requirement (BSCR) which together with operational risk yields SCR for the entire company. Conditions and assumptions are summarized in Table 1. Correlations between the variables under the non-life and market risk modules are needed and also between non-life, credit and market risk one level above. There is also a correlation for the relationship between BSCR and operational risk at the top. The values quoted are with one small modification (see below) taken from Commission Delegated Regulation (2014) and were obtained by statistical studies of the European insurance industry. Skewness parameters are moderate except for catastrophe, credit and operational risk where values are much higher. Those for credit and operational risk are taken from Sandström (2011), see p 240. Standard deviations reflect the volume of company business with three strategies $\mathrm{A}, \mathrm{B}$ and $\mathrm{C}$. The first two assume the same insurance portfolios, but differ in investments with B much heavier in equity and nothing in property. In strategy $\mathrm{C}$ catastrophe insurance has been removed, and the premium/reserve component doubled.

Bottom-up modelling is needed to validate the approximations against nearly-exact SCR's obtained by Monte Carlo. This means a joint model for the input variables in Table 1 which are Premium/Reserve, Cat, Lapse, Credit risk, Equity, Bonds/Cash and Property. Consider first the model in Section 2.3 where dependence was defined by the correlation matrix of the normal variates in (5). The aim is to 


\begin{tabular}{|cccccc|cc|cccccc|}
\hline \multicolumn{10}{|c|}{ Operational risk: $\sigma=0.05, \gamma=4.5$ with maximum correlation with BSCR } \\
Correlations for Non-life, Credit, Market: $\rho_{12}=0.50, \rho_{13}=0.25, \rho_{23}=0.25$ \\
Non-life \\
Cat
\end{tabular}

Table 1 Scenarios for the experiments in Section 5. Company strategies $A, B$ and $C$ are defined by the standard deviations $\sigma$ with changes from strategy $A$ in bold face.

calibrate it so that all the correlations in Table 1 are reproduced. Those between the Non-life, Credit and Market modules at the second level is then a an obstacle that can't be overcome in general (see Filipovic, 2009), but in the present case it could by selecting cross-correlations between variables in different modules numerically, aided by Monte Carlo (one million simulations). Operational risk has in Solvency II correlation 1 with BSCR, a value beyond reach for log-normals, and the maximum that could be achieved was selected in its place.

A similar, copula-based model is also needed. It should, like the other one, recreate the correlations in Table 1 to promote insight into the impact of discrepant treatments of tail-dependency. Multiple, hierarchical copula modelling is treated in great generality in Bedford and Cooke (2002) with Aas, Czabo, Frigessi and Bakken (2009) and Savelli and Clemente (2011) contributions in insurance. In Savelli and Clemente (2011) the aggregate distribution has been obtained by assuming a log-normal distribution and by using Hierarchical Copula without any approximation, as both marginal distributions and copula have been generated by Monte Carlo methods.

A convenient approach in the present context was proposed by McNeil (2008) who pointed out that the Clayton copula (16) can be used recursively when dealing with trees like the one in Figure 1. His technique opens up a huge number of possibilities, and by trial and error aided by Monte Carlo 


\begin{tabular}{|l|ccccc|cc|}
\hline Stra- & \multicolumn{5}{|c|}{ Dependence through correlations } & \multicolumn{3}{|c|}{ Clayton dependence } \\
tegy & 'Gaussian' & 'Solvency II' & Cornish-Fisher & Log-normal & Exact & Log-normal & Exact \\
A & 5.63 & 8.04 & 11.47 & 7.89 & $\mathbf{7 . 5 9}$ & 8.31 & $\mathbf{8 . 5 6}$ \\
B & 6.94 & 9.24 & 13.29 & 8.84 & $\mathbf{8 . 6 3}$ & 9.32 & $\mathbf{9 . 7 2}$ \\
C & 6.58 & 7.11 & 7.47 & 6.99 & $\mathbf{6 . 9 6}$ & 7.11 & $\mathbf{7 . 3 1}$ \\
\hline
\end{tabular}

Table 2 SCR approximations and nearly exact values under the conditions and company strategies in Table 1. Dependence model in terms of correlations on the left and the hierarchical Clayton copula in Appendix $B$ on the right.

(one million simulations) we arrived at the iterative Clayton/McNeil scheme in Appendix B which produces correlations very close to those in Table 1.

SCR evaluations are summarized in Table 2. The left hand side where dependence is through ordinary correlations confirms the picture in Figure 2 with the log-normal approximation quite accurate with an error at $4-5 \%$ on the conservative side. 'Solvency II' is a good deal less accurate whereas the Cornish-Fisher results are up to $60 \%$ too high. The difference between Scenarios A and B had little impact on error, but in Scenario C with no catastrophe insurance at all every method worked except Cornish-Fisher. Moving on to Clayton dependence on the right, one striking feature is how much assessments have gone up. Replacing correlation matrices with a comparable tail dependence model lead to increases of $30-40 \%$ in the SCR's. The log-normal approximation, when fed the correct model, is still accurate.

\section{Concluding discussion}

The log-normal distribution with moment matching up to order three provides fair accuracy and preserves much of the simplicity in the Solvency II standard formula (see, Braun, Schmeiser and Schreiber, 2015 and Eling and Pankoke, 2014). Errors were largest in unbalanced situations with strong skewness diversification between variables, and this may well be typical. Approximation based 
on the log-normal is superior to a non-parametric approach of the Cornish-Fisher type where the weakness in how skewness is aggregated can't be easily rectified when variables are dependent. The log-normal family is a first choice when moving out of the pure Gaussian since it is so frequently cited in Solvency II literature, but it was not examined whether it is a better vehicle than other heavy-tailed distributions such as Gamma, Burr or inverse Gaussian which can be developed in much the same way. It may be worth a study: Do some families lead to methods more robust towards model error than others, and if erring on the conservative side is what we want, should this influence the family selected?

Simulation was avoided when the log-normal method was constructed from dependent Gaussian vectors, but that implies a certain, narrow view on tail dependence, and copulas may adapt better to reality. This is an issue of some importance since SCR evaluations are sensitive towards the type of tail dependence as the example in Section 5 demonstrated. The log-normal approximation was still accurate, and the Monte Carlo (which is now required) does not add too much complexity, but can the simplicity be maintained in other respects? Specifying dependence in terms of correlations is straightforward whereas copulas may appear less immediate, yet hard to avoid if the $99.5 \%$ security advocated by the European regulatory authorities is taken seriously. The correlation matrices of Solvency II of today must then be taken out and replaced by copulas.

Funding: MG work was supported by the Spanish Ministry/ERDF [ECO2013-48326-C2-1 / ECO201676203-C2-2-P] and ICREA Academia. No conflict of interest exists.

\section{References}

Aas, K., Czado, C., Frigessi, A. and Bakken, H. (2009). Pair-copula constructions of multiple de- 
pendence. Insurance: Mathematics \& Economics, 44, 182-198..

Alm, J. (2015). A simulation model for calculating solvency capital requirements for non-life insurance risk. Scandinavian Actuarial Journal, 107-123.

Asmussen, S., Blanchet, J., Juneja, S. and Rojas-Nandyapa, L. (2011). Efficient simualtion of tail probabilities of correlated log-normals. Annals of Operations Research, 189, 5-23.

Bedford, T. and Cooke, R.M. (2002). Probability density decomposition for conditionally dependent random variables modelled by vines. Annals of Mathematical Artificial Intelligence 32, 215-268.

Berggren, F. (2005). An error bound for moment matching methods of log-normal sum distributions. European Transactions of Telecommunication, 16, 573-577.

Bermudez, L., Ferri, A. and Guillen, M. (2013). A correlation sensitivity analysis of non-life underwriting risk in Solvency II. Astin Bulletin, 41, 23-37.

Bølviken, E. (2014). Computation and modelling in insurance and finance. Cambridge University Press, Cambridge.

Braun, A., Schmeiser, H. and Schreiber, F. (2015). Market risk standard formula: How credible is the proclaimed ruin probability? Journal of Insurance Issues, 38, 1-30.

Clemente,G.P. and Savelli, N. (2015). Internal model techniques of premium and reserve risk for nonlife insurers. Mathematical methods in Economics and Finance (A), 21-34.

Commission Delegated Regulation (2015). In the Official Journal of The European Union. Available as http://eur-lex.europa.eu/legal-content/EN/TXT/?uri=CELEX

Dufresne, D. (2004). The log-normal application in financial and other applications. Advances Applied Probability, 36, 747-773.

Eling, M. (2012). Fitting insurance claims to skewed distributions: Are the skew-normal and skewstudent good models?. Insurance: Mathematics and Economics, 51(2), 239-248.

Eling, M. and Pankoke, D. (2014). Basis risk procycliciality and systemic risk in the Solvency II equity 
risk module. Journal of Insurance Regulation, 33, 1-39.

EIOPA (2014). The underlying assumptions in the standard formula for the Solvency III capital requirement calculatiuon. Available as https://eiopa.europa.eu/Publicationa/Standards/EIOPA-14322_Underlying_Assumptions.pdf.

Fenton, L.F. (1960). The sum of log-normal probability distributions in scatter transmissions systems. IRE Transactions on Communications Systems, CS-8, 57-67.

Filipovic, D. (2009). Multi-level risk aggregation. Astin Bulletin, 39, 565-575.

Henriksen, P. (2008). Lognormal moment matching and pricing of basket options. Statistical Research Report, Department of Mathematics, University of Oslo.

McNeil, A.L. (2008). Sampling nested Archimedean Copulas. Journal of Statistical Computation and Simulation, 78, 567-581.

Mehta, N.B, Wu, J., Molisch, A. and Zhang, J. (2007). Approximating a sum of random variables with a lognormal. IEEE Transactions on Wireless Communication, 6, 2690-2699.

Pfeifer, P. and Strassburger, D. (2008). Solvency II: Stability problems with the SRC aggregation formula. Scandinavian Actuarial Journal, 61-77, with Corrigendum (2009), 315.

Sandström, A. (2007). Solvency II: Calibration for skewness. Scandinavian Actuarial Journal,126-134. Sandström A. (2011). Handbook of solvency for actuaries and risk managers. Chapman \& Hall/CRC, Boca Raton, Lousiana

Savelli, N. and Clemente, G.P. (2011). Hierarchical structures in the aggregation of premium risk for insurance underwriting. Scandinavian Actuarial Journal, 193-213.

Senaratne, D. and Tellambura, C. (2009). Numerical computation of the lognormal sum distribution. 2009 IEEE Telecommunications Conference (GLOBECOM2009), 1-6.

Wilkinson, J. (1934). Bell Telephone Labs. Unpublished.

Zhang, Q.T. and Song, S.H. (2008). A systematic procedure for accurately approximating lognormal- 
sum distribution. IEEE Transactions on Vehicular Technology, 57, 663-666.

\section{Appendix A. Skewness of the sum of log-normals.}

It follows from (8) that $\gamma_{s}=E\left(S^{3}\right) / \sigma_{s}^{3}$ can be written

$$
\gamma_{s}=B_{1}+2 B_{2}+6 B_{3}
$$

where

$$
B_{1}=\frac{1}{\sigma_{s}^{3}} \sum_{i} E\left(X_{i}^{3}\right), \quad B_{2}=\frac{1}{\sigma_{s}^{3}} \sum_{i \neq j} E\left(X_{i}^{2} X_{j}\right), \quad B_{3}=\frac{1}{\sigma_{s}^{3}} \sum_{i<j<k} E\left(X_{i} X_{j} X_{k}\right) .
$$

The skewness expression (6) right yields

$$
\frac{1}{\sigma_{s}^{3}} E\left(X_{i}^{3}\right)=\frac{\sigma_{i}^{3}}{\sigma_{s}^{3}}\left(e^{\tau_{i}^{2}}+2\right)\left(e^{\tau_{i}^{2}}-1\right)^{1 / 2}=\alpha_{i}^{3}\left(e^{\tau_{i}^{2}}+2\right)\left(e^{\tau_{i}^{2}}-1\right)^{2}
$$

with $\alpha_{i}$ as in (10) left. Hence $B_{1}$ is the first sum on the right in (9).

To verify that the other two sums coincide with $B_{2}$ and $B_{3}$ we need $\rho_{i j}=\operatorname{cor}\left(X_{i}, X_{j}\right)$ which is related to $\omega_{i j}=\operatorname{cor}\left(\varepsilon_{i}, \varepsilon_{j}\right)$ through

$$
\rho_{i j}=\frac{e^{\omega_{i j} \tau_{i} \tau_{j}}-1}{\left\{\left(e^{\tau_{i}^{2}}-1\right)\left(e^{\tau_{j}^{2}}-1\right)\right\}^{1 / 2}} \quad \text { or } \quad e^{\omega_{i j} \tau_{i} \tau_{j}}=1+\rho_{i j} \sqrt{\left(e^{\tau_{i}^{2}}-1\right)\left(e^{\tau_{j}^{2}}-1\right)}=\beta_{i j}
$$

with $\beta_{i j}$ as in (10). Inserting for $X_{i}$ and $X_{j}$ yields

$$
\begin{aligned}
B_{2} & =\sum_{i \neq j} \alpha_{i}^{2} \alpha_{j} E\left(\left(e^{-\tau_{i}^{2} / 2+\tau_{i} \varepsilon_{i}}-1\right)^{2}\left(e^{-\tau_{j}^{2} / 2+\tau_{j} \varepsilon_{j}}-1\right)\right) \\
& =\sum_{i \neq j} \alpha_{i}^{2} \alpha_{j} E\left(e^{-\tau_{i}^{2}-\tau_{j}^{2} / 2+2 \tau_{i} \varepsilon_{i}+\tau_{j} \varepsilon_{j}}-2 e^{-\tau_{i}^{2} / 2-\tau_{j}^{2} / 2+\tau_{i} \varepsilon_{i}+\tau_{j} \varepsilon_{j}}+e^{-\tau_{j}^{2} / 2+\tau_{j} \varepsilon_{j}}-\left(e^{-\tau_{i}^{2} / 2+\tau_{i} \varepsilon_{i}}-1\right)^{2}\right) .
\end{aligned}
$$

The expectations of the four terms inside the outer parenthesis can be calculated by the elementary formula for the mean of log-normals which leads to

$$
E\left(e^{-\tau_{i}^{2}-\tau_{j}^{2} / 2+2 \tau_{i} \varepsilon_{i}+\tau_{j} \varepsilon_{j}}\right)=e^{\tau_{i}^{2}+2 \omega_{i j} \tau_{i} \tau_{j}}, \quad E\left(e^{-\tau_{i}^{2} / 2-\tau_{j}^{2} / 2+\tau_{i} \varepsilon_{i}+\tau_{j} \varepsilon_{j}}\right)=e^{\omega_{i j} \tau_{i} \tau_{j}}
$$




$$
E\left(e^{-\tau_{j}^{2} / 2+\tau_{j} \varepsilon_{j}}\right)=1 \quad \text { and } \quad E\left(e^{-\tau_{i}^{2} / 2+\tau_{i} \varepsilon_{i}}-1\right)^{2}=e^{\tau_{i}^{2}}-1
$$

But now some straightforward calculations imply that

$$
B_{2}=\sum_{i \neq j} \alpha_{i}^{2} \alpha_{j}\left\{e^{\tau_{i}^{2}}\left(e^{2 \omega_{i j} \tau_{i} \tau_{j}}-1\right)-2\left(e^{\omega_{i j} \tau_{i} \tau_{j}}-1\right)\right\}
$$

which becomes the second sum in (9) when $e^{\omega_{i j} \tau_{i} \tau_{j}}$ is replaced by $\beta_{i j}$.

Then there is $B_{3}$ which after inserting for $X_{i}, X_{j}$ and $X_{k}$ equals

$$
B_{3}=\sum_{i<j<k} \alpha_{i} \alpha_{j} \alpha_{k} E\left(\left(e^{-\tau_{i}^{2} / 2+\tau_{i} \varepsilon_{i}}-1\right)\left(e^{-\tau_{j}^{2} / 2+\tau_{j} \varepsilon_{j}}-1\right)\left(e^{-\tau_{k}^{2} / 2+\tau_{k} \varepsilon_{k}}-1\right)\right)
$$

The expectation of the product is derived by multiplying out the the three factors so that it becomes a sum of eight terms, i.e.

$$
\begin{gathered}
e^{-\tau_{i}^{2} / 2-\tau_{j}^{2} / 2-\tau_{k}^{2} / 2+\tau_{i} \varepsilon_{i}+\tau_{j} \varepsilon_{j}+\tau_{k} \varepsilon_{k}}-e^{-\tau_{i}^{2} / 2-\tau_{j}^{2} / 2+\tau_{i} \varepsilon_{i}+\tau_{j} \varepsilon_{j}}-e^{-\tau_{i}^{2} / 2-\tau_{k}^{2} / 2+\tau_{i} \varepsilon_{i}+\tau_{k} \varepsilon_{k}} \\
-e^{-\tau_{j}^{2} / 2-\tau_{k}^{2} / 2+\tau_{j} \varepsilon_{j}+\tau_{k} \varepsilon_{k}}+e^{-\tau_{i}^{2} / 2+\tau_{i} \varepsilon_{i}}+e^{-\tau_{j}^{2} / 2+\tau_{j} \varepsilon_{j}}+e^{-\tau_{k}^{2} / 2+\tau_{k} \varepsilon_{k}}-1,
\end{gathered}
$$

and the formula for the mean of log-normal variables now yields

$$
B_{3}=\sum_{i<j<k} \alpha_{i} \alpha_{j} \alpha_{k}\left(e^{\omega_{i j} \tau_{i} \tau_{j}+\omega_{i k} \tau_{i} \tau_{k}+\omega_{j k} \tau_{j} \tau_{k}}-e^{\omega_{i j} \tau_{i} \tau_{j}}-e^{\omega_{i k} \tau_{i} \tau_{k}}-e^{\omega_{j k} \tau_{j} \tau_{k}}+1+1+1-1\right)
$$

which becomes the third sum in $(9)$ when $e^{\omega_{i j} \tau_{i} \tau_{j}}=\beta_{i j}$ is inserted everywhere.

\section{Appendix B. A hierarchical Clayton copula.}

Bottom-up models for the simplified insurance company in Figure 1 must be constructed through Premium/Reserve (variable $\left.X_{1}\right)$, Cat $\left(X_{2}\right)$, Lapse $\left(X_{3}\right)$, Credit risk $\left(X_{4}\right)$, Equity $\left(X_{5}\right)$, Bonds/Cash $\left(X_{6}\right)$ and Property $\left(X_{7}\right)$. Their percentile functions transform them to uniforms $U_{1}, \ldots, U_{7}$ for which we seek a copula with higher dependence at the right tail that on average. It should reproduce the cor-

relations in Table 1, including those between the non-life, credit risk and market risk modules. Using 
the Clayton copula iteratively as in McNeil (2008) provides all of this. The mixing relationship (16) is a central building block, and we also need the alternative form of that model which specifies the second uniform as

$$
U_{2}=\left\{1+U_{1}^{-\theta}\left(V^{-\theta /(1+\theta)}-1\right)\right\}^{-1 / \theta}
$$

where $V$ is uniform and independent of $U_{1}$; consult Chapter 6 in Bølviken (2014). In a chain of applications where the aim is to capture correlations between groups of variables it does matter which version is used.

The following set of commands defines the model for $U_{1}, \ldots, U_{7}$ in Section 5 . Note the uniforms $U_{\mathrm{nl}}, U_{\mathrm{cr}}, U_{\mathrm{ma}}$ assigned the Non-life, Credit risk and Market risk modules to create the approriate crosscorrelations between them. They are generated through a separate copula construction at the start of the algorithm. Gamma variables with mean 1 and shape $1 / \theta$ are written $\operatorname{Gamma}(1 / \theta)$ and their percentile function $G(u ; 1 / \theta)$. This yields when $\sim$ denote drawings:

$$
\begin{aligned}
& \text { Uniforms for cross-dependencies between modules } \\
& U_{\text {nl }} \sim \text { uniform, } \quad V_{\text {cr }} \sim \text { uniform, }
\end{aligned}
$$

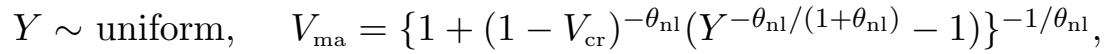

$$
\begin{aligned}
& U_{\text {cr }}=\left\{1+U_{\mathrm{nl}}^{-\theta_{\mathrm{cr}}}\left(V_{\mathrm{cr}}^{-\theta_{\mathrm{cr}} /\left(1+\theta_{\mathrm{cr}}\right)}-1\right)\right\}^{-1 / \theta_{\mathrm{cr}}}, \\
& U_{\mathrm{ma}}=\left\{1+U_{\mathrm{nl}}^{-\theta_{\mathrm{ma}}}\left(V_{\mathrm{ma}}^{-\theta_{\mathrm{ma}} /\left(1+\theta_{\mathrm{ma}}\right)}-1\right)\right\}^{-1 / \theta_{\mathrm{ma}}} \text {. } \\
& \text { Uniforms for the non-life module } \\
& Z=G^{-1}\left(U_{\mathrm{nl}} ; 1 / \theta_{1}\right), \quad V_{1} \sim \text { uniform }, \quad V_{2} \sim \text { uniform } \\
& U_{i}=\left(1-\theta_{1} \log \left(V_{i}\right) / Z\right)^{-1 / \theta_{1}}, i=1,2, \quad U_{3} \sim \text { uniform. } \\
& U_{4}=U_{\text {cr }} \text {. } \\
& \text { Uniform for the credit risk module } \\
& \text { Uniforms for the market risk module } \\
& Z_{i} \sim \operatorname{Gamma}\left(1 / \theta_{i}\right), \quad U_{i}=\left(1-\theta_{i} \log \left(U_{\mathrm{ma}}\right) / Z_{i}\right)^{-1 / \theta_{i}}, i=5,6,7 \text {. }
\end{aligned}
$$

The model was found be trial and error and reproduce the correlations in Table 1 not far from exactly if the parameters are $\theta_{\mathrm{nl}}=0.17, \theta_{\mathrm{cr}}=1.87, \theta_{\mathrm{ma}}=0.82, \theta_{1}=0.328, \theta_{5}=0.34, \theta_{6}=1.65, \theta_{7}=0.34$. 